\title{
Optical analysis of ball-on-ring mode test rig for oil film thickness measurement
}

\author{
Yaoguang ZHANG, Wenzhong WANG ${ }^{*}$, Shengguang ZHANG, Ziqiang ZHAO \\ School of Mechanical Engineering, Beijing Institute of Technology, Beijing 100081, China \\ Received: 16 July 2016 / Revised: 26 September 2016 / Accepted: 26 October 2016 \\ (C) The author(s) 2016. This article is published with open access at Springerlink.com
}

\begin{abstract}
There are few experimental results available on film thickness at speeds above $5 \mathrm{~m} / \mathrm{s}$ and they are almost all based on the optical ball-on-disc test rig. In contrast to the contacts in a rolling bearing, in which the lubricant in the oil reservoir distributes symmetrically, ball-on-disc contact shows asymmetry of lubricant distribution due to centrifugal effects. In order to closely imitate the contact occurring between the ball and the outer ring of a ball bearing, this study proposes an experimental model based on ball-on-glass ring contact. An optical matrix method is used to analyze the optical system, which is composed of a steel ball-lubricant-chromium-coated glass ring. Based on the optical analysis, the measurement system is improved in order to obtain a high quality interference image, which makes it possible to measure the film thickness at high-speeds conditions.
\end{abstract}

Keywords: film thickness measurement; optical interference; optical matrix; rolling bearing; high speed

\section{Introduction}

Over the past few decades, the measurement of lubricant film thickness has caught considerable attention of many researchers in the elastohydrodynamic lubrication (EHL) community. Given that the film thickness is extremely thin in the Hertzian contact region, an optical interferometry-based method can measure the film thickness accurately. This is shown by the experimental investigations of EHL, owing to a comparable magnitude between optical light wavelength and lubricant film thickness. In the 1960s, Gohar and Cameron first applied optical interferometry to the measurement of oil film thickness in EHL, capturing the first classical interference image with a rotating steel ball loaded against a glass plate [1]. They then gave the results both in point and line contacts in their following works [2]. The subsequent development and improvement of this technique were focused on the lower limit of measurable thickness and higher resolution. The technique used by Spikes

* Corresponding author: Wenzhong WANG.

E-mail: wangwzhong@bit.edu.cn and his group could measure films down to less than $5 \mathrm{~nm}$ at very low speeds, which combined a spacer layer with spectrometric analysis [3]. Based on this technique, an imaging system was developed to profile the film in the EHL contacts [4], and later extended to obtain the film thickness distribution in the contact area by combining the spacer layer technique with the color image analysis [5]. Aiming for accurate determination of lubricant film thickness distribution, Hartl's group developed an experimental technique that combined chromatic interferometry with a computer image processing method. They obtained a three-dimensional distribution of the EHL film in a range of $60-800 \mathrm{~nm}$ with high resolution and accuracy [6]. This colorimetric interferometry used by the same group allowed the study of ultra-thin lubrication films down to $1 \mathrm{~nm}$ [7]. The technique, based on monochromatic interferometry, also had a low minimum measurable thickness and high resolution. The relative optical interference intensity method proposed by Luo et al. determined the film thickness by the interference intensity of the point in the contact region, with the location of this intensity between the 


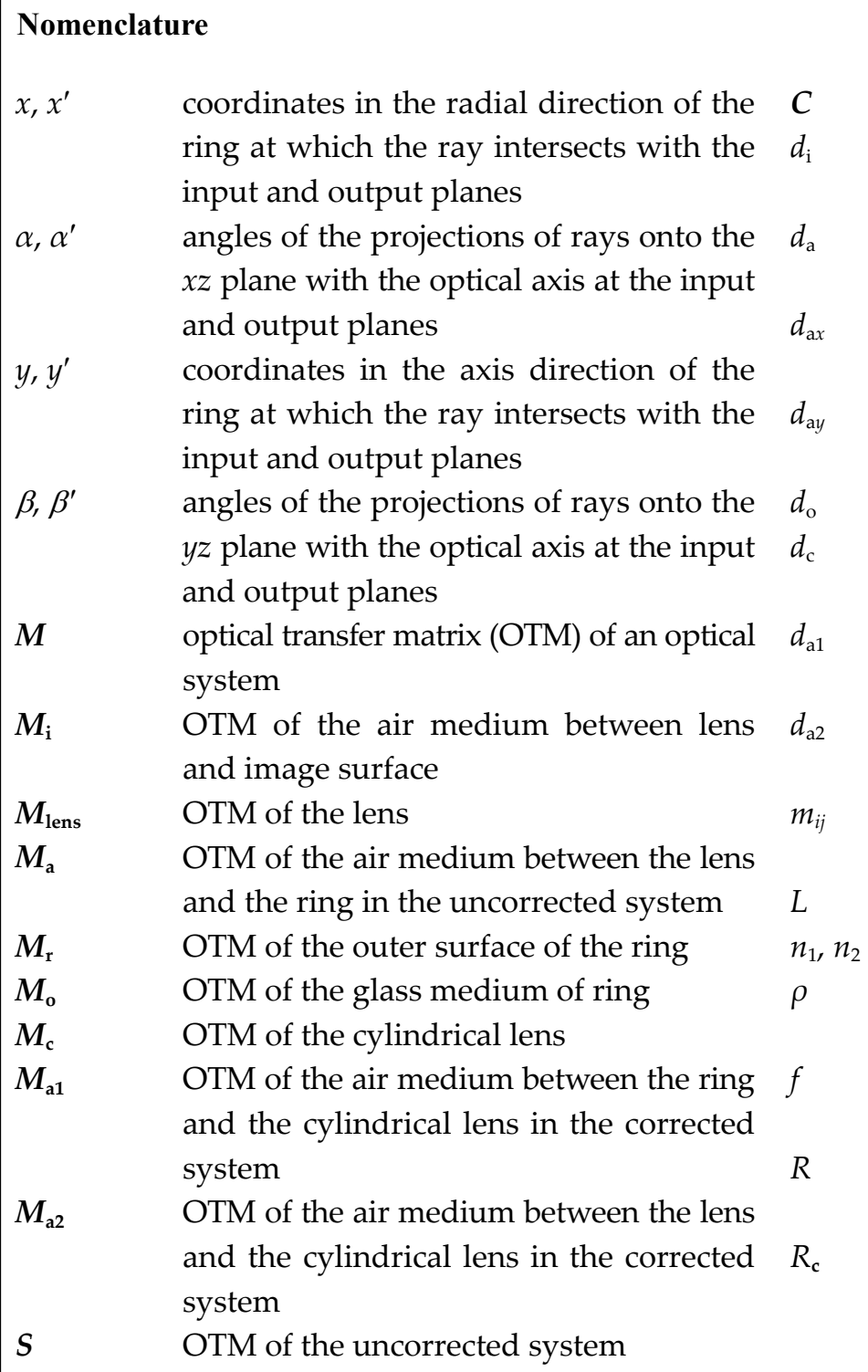

\section{OTM of the corrected system}

distance between the image surface and the lens

distance between the lens and the ring in the uncorrected system

$d_{\mathrm{a}}$ for the object-image relationship in the $x$ direction to hold

$d_{\mathrm{a}}$ for the object-image relationship in the $y$ direction to hold

thickness of the glass ring

thickness of the cylindrical lens, the curvature of which is ignored

distance between the ring and the cylindrical lens in the corrected system

distance between the lens and the cylindrical lens in the corrected system

the element at the $i^{\text {th }}$ row and $j^{\text {th }}$ column of $\boldsymbol{M}$

width of the homogeneous medium layer refractive index of glass $\mathrm{K} 9$ and air radius of the sphere, $\rho>0$ for convex (center of curvature after interface)

focal length of lens, $f>0$ for convex/positive (converging) lens

$R \quad$ radius of the ring, $R>0$ for convex (center of curvature after interface)

radius of the cylindrical lens, $R_{\mathrm{c}}>0$ for convex (center of curvature after interface) darkest and brightest fringes. The intensity came from the interference of two reflected beams [8]. Considering the effects of multi-beam interference and the optical absorption of metals, Guo and Wong [9] developed a multi-beam intensity-based technique with a resolution of $1 \mathrm{~nm}$ and a minimum measurable thickness of $1 \mathrm{~nm}$.

Experimental studies to measure the film thickness in EHL are mainly based on abovementioned optical interferometry mechanism. Most research studies have been focused on the lubrication performance at speeds lower than $5 \mathrm{~m} / \mathrm{s}$. Only a few experimental studies have explored the film thickness behavior at speeds higher than $5 \mathrm{~m} / \mathrm{s}$. The limited experimental results at very high speeds show that elastohydrodynamic films are thinner than that predicted by classical theoretical models, such as the Hamrock and Dowson equation. Hili [10] concluded that the inlet shear heating has a significant effect on the behavior of film thickness at very high speeds up to $20 \mathrm{~m} / \mathrm{s}$. Liang et al. [11] studied the behavior of starved EHL contacts at high speeds up to $42 \mathrm{~m} / \mathrm{s}$ with ball-disc contact, the results of which indicated that centrifugal force significantly affects the starved behavior of the lubricant film.

However, almost all published experimental studies on EHL film thickness measurement of the point 
contacts are based on ball-on-disc or ball-on-plate contacts, where the speed distribution in the ballon-disc is different from the speed distribution in a rolling bearing. Compared to a rolling bearing, where the centrifugal force is perpendicular to the contact surface, the centrifugal force in the ball-on-disc contact is parallel to the disc face, which drags the oil out of the contact region. This may increase the oil supply, and also lead to the interference image haziness as mentioned in Liang's study [11].

This paper reports a new ball-on-ring model for the measurement of oil film thickness, which may be suitable to imitate contact conditions occurring in a high-speed ball bearing. This model avoids the uneven distribution of lubricant between the two sides of the "raceway", which occurs in the ball-on-disc contact because centrifugal force pulls the lubricant away from the contact region. Owing to unexpected optical refraction at the outer cylindrical surface of the ring, the initial interference images are fuzzy and out-offocus. To solve the problem, the matrix optics method [12] is used to analyze the optical system, and a correction approach is proposed to modify the optical path. The preliminary experimental results prove the feasibility of the ball-on-ring model in the measurement of oil film thickness.

\section{Optical analysis of the ball-on-ring model}

The test rig, as shown in Fig. 1, was developed to measure the film thickness of the lubricant in the point contact region. The test rig imitates a rolling bearing in the form of a steel ball in contact with a ring made of $\mathrm{K} 9$ optical glass. A supporting system containing four well-adjusted small rolling bearings
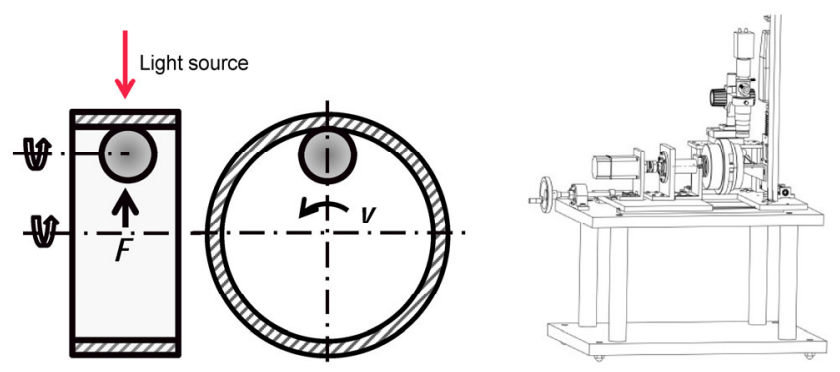

Fig. 1 Schematic of the optical measurement system based on the ball-on-ring model. underneath the ball ensures that the ball rotates around an axis parallel to the glass ring's central axis. The inner surface of the glass ring is coated with a very thin, semi-reflective layer of chromium with the reflectivity of about 20 percent, thus when the incident light has reflected from the steel ball and refracted at the chromium, two interfering beams have approximately equal intensities, which enables the interference to occur and the film thickness to be determined. A laser device is used to provide light with good coherence for optical interference image. The interference images are magnified using a coaxial microscope and then captured by a charge coupled device (CCD), which is coupled with the microscope. Figure 2 shows a set of interference images captured by the newly developed test rig at different object distances, ranging from far to near the ring. It was found that the resultant interference images were not clear and showed blurring and ghosting. Owing to the haziness of the interference image, it could not be used to determine the film thickness. The reason for the image haziness should be explored in order to improve the quality of the interference images. Therefore, an optical model for the test rig was developed to conduct optical analysis based on the following optical matrix method.

The optical transfer matrix provides an effective method of analyzing the cause for the fuzziness in the interference images shown in Fig. 2. Figure 3 gives the definition of a ray, where $h_{x}$ and $h_{y}$ are the coordinates of the point of intersection at which the ray intersects the reference plane, $\alpha$ is the angle between the projection of the ray in the $x z$ plane and $z$ axis, and $\beta$ is the angle between the projection of the ray in the $y z$ plane and $z$ axis. The propagation of a paraxial ray through a system of centered lenses can be written in the matrix form:

$$
\left[\begin{array}{l}
x^{\prime} \\
\alpha^{\prime} \\
y^{\prime} \\
\beta^{\prime}
\end{array}\right]=\boldsymbol{M}\left[\begin{array}{l}
x \\
\alpha \\
y \\
\beta
\end{array}\right]
$$

where the matrix $M$ is the optical transfer matrix.

Figure 4(a) shows the optical model of the ballon-ring test rig system. Assuming that the interference images emerge at the inner surface of the ring, and the contact region of the ring is the plane (the depth 


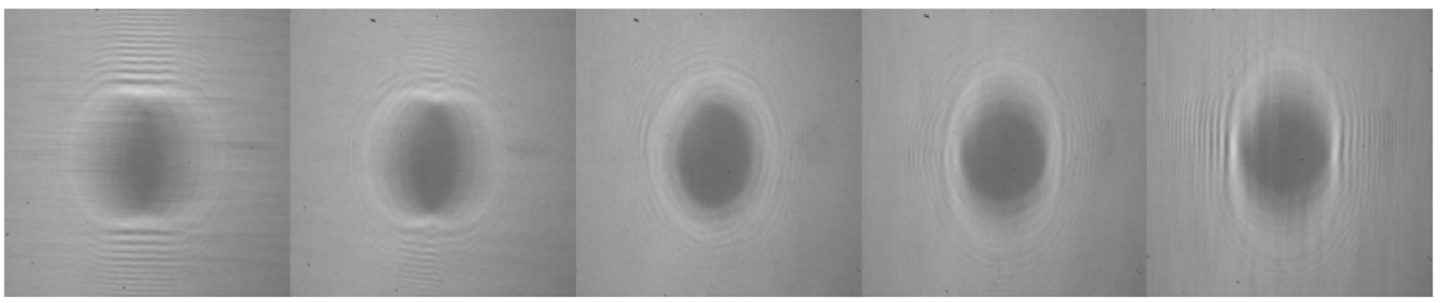

Fig. 2 A series of fuzzy images with varying distances between the microscope and the ring.

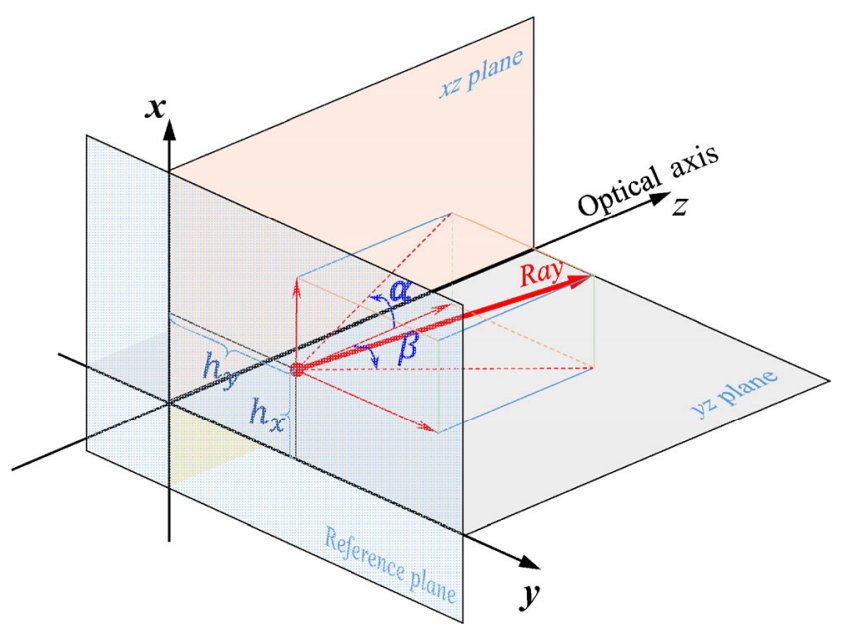

Fig. 3 Description of a ray. of the image region in the optical axis direction is much shallower than the depth of the field of the microscope), the optical system of the ball-on-ring test rig can be simplified as an ideal optical system consisting of a cylindrical interface, a thin lens, and several homogeneous medium layers as shown in Fig. 4(b). It should be noted that we use a thin lens to represent the microscope, because the microscope consists of lenses with strict axial symmetry and has the same refraction ability for the rays in different directions, then it will has the same function as a thin lens to converge the light ray from an object point to an image point.

For simplicity and clarity, we first consider the propagation of light in the $x z$ plane.

(a)
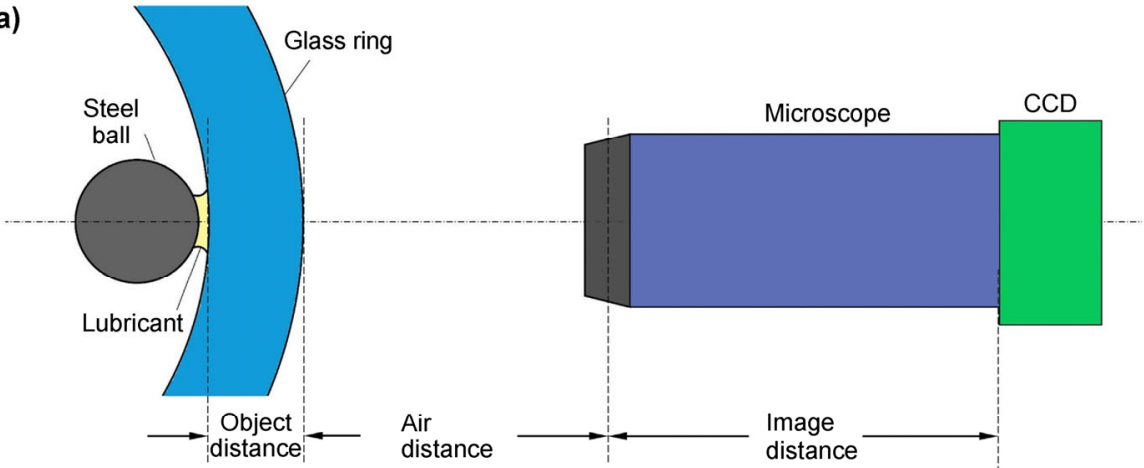

(b)

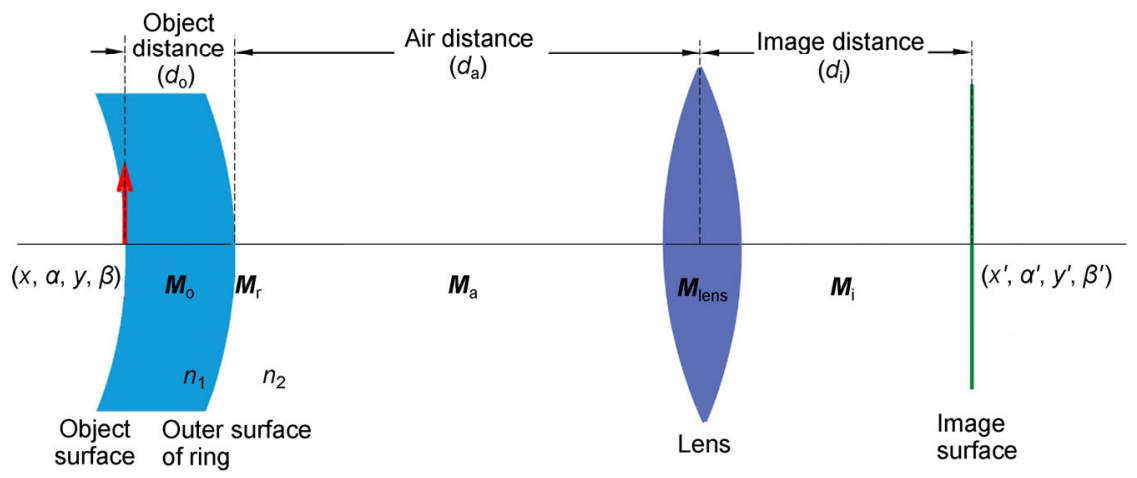

Fig. 4 Optical system of the ball-on-ring test rig. 
Light traveling from one surface to another in a homogeneous medium layer with thickness $L$ obeys the following equation:

$$
\left[\begin{array}{l}
x^{\prime} \\
\alpha^{\prime}
\end{array}\right]=\boldsymbol{M}_{x}\left[\begin{array}{l}
x \\
\alpha
\end{array}\right]=\left[\begin{array}{ll}
1 & L \\
0 & 1
\end{array}\right]\left[\begin{array}{l}
x \\
\alpha
\end{array}\right]
$$

Light traveling through a thin lens with a focal length $f$ obeys the following equation:

$$
\left[\begin{array}{l}
x^{\prime} \\
\alpha^{\prime}
\end{array}\right]=\boldsymbol{M}_{x}\left[\begin{array}{l}
x \\
\alpha
\end{array}\right]=\left[\begin{array}{cc}
1 & 0 \\
-\frac{1}{f} & 1
\end{array}\right]\left[\begin{array}{l}
x \\
\alpha
\end{array}\right]
$$

Light traveling through a curved interface between two mediums with refractive indexes $n_{1}$ and $n_{2}$ (light travels from $n_{1}$ to $n_{2}$ ) obeys the following equation:

$$
\left[\begin{array}{c}
x^{\prime} \\
\alpha^{\prime}
\end{array}\right]=\boldsymbol{M}_{x}\left[\begin{array}{l}
x \\
\alpha
\end{array}\right]=\left[\begin{array}{cc}
1 & 0 \\
\frac{n_{1}-n_{2}}{n_{2} \rho} & \frac{n_{1}}{n_{2}}
\end{array}\right]\left[\begin{array}{l}
x \\
\alpha
\end{array}\right]
$$

where $\rho$ is the radius of the curved interface.

Similarly, the propagation of light in the $y z$ plane can be obtained. Then, the above cases are extended to three dimensional situations. The propagation of light can be described as follows:

$$
\left[\begin{array}{c}
x^{\prime} \\
\alpha^{\prime} \\
y^{\prime} \\
\beta^{\prime}
\end{array}\right]=\left[\begin{array}{cccc}
\boldsymbol{M}_{x} & 0 & 0 \\
0 & 0 & & 0 \\
0 & 0 & \boldsymbol{M}_{y}
\end{array}\right]\left[\begin{array}{c}
x \\
\alpha \\
y \\
\beta
\end{array}\right]
$$

When there is both a homogeneous medium layer and a thin lens, $\boldsymbol{M}_{x}=\boldsymbol{M}_{y}$. However, for a ray traveling through a cylindrical interface, the refractions of the ray at the cylindrical interface in the $x z$ plane and the $y z$ plane are different due to the different radii; therefore, from Eq. (4), Eq. (5) becomes

$$
\left[\begin{array}{c}
x^{\prime} \\
\alpha^{\prime} \\
y^{\prime} \\
\beta^{\prime}
\end{array}\right]=\left[\begin{array}{cccc}
1 & 0 & 0 & 0 \\
\frac{n_{1}-n_{2}}{n_{2} \rho} & \frac{n_{1}}{n_{2}} & 0 & 0 \\
0 & 0 & 1 & 0 \\
0 & 0 & 0 & \frac{n_{1}}{n_{2}}
\end{array}\right]\left[\begin{array}{c}
x \\
\alpha \\
y \\
\beta
\end{array}\right]
$$

Therefore, the optical transfer matrix of the simplified optical system for the ball-on-ring model, as shown in Fig. 4(b), can be represented in terms of matrix multiplication as follows:

$$
\boldsymbol{S}=\boldsymbol{M}_{\mathrm{i}} \boldsymbol{M}_{\text {lens }} \boldsymbol{M}_{\mathrm{a}} \boldsymbol{M}_{\mathrm{r}} \boldsymbol{M}_{\mathrm{o}}
$$

where $\boldsymbol{M}_{\mathrm{o}}$ is the optical transfer matrix when light travels in the glass ring, $\boldsymbol{M}_{\mathrm{r}}$ is the transfer matrix when light travels through the outer surface of the glass ring, $\boldsymbol{M}_{\mathrm{a}}$ is the transfer matrix when light travels in the air between the glass ring and the lens, $\boldsymbol{M}_{\mathrm{lens}}$ is the transfer matrix when light travels through the lens, and $\boldsymbol{M}_{\mathrm{i}}$ is the transfer matrix when light travels in the air between the lens and the CCD. They are described as follows:

$\boldsymbol{M}_{\mathrm{i}}=\left[\begin{array}{cccc}1 & d_{\mathrm{i}} & 0 & 0 \\ 0 & 1 & 0 & 0 \\ 0 & 0 & 1 & d_{\mathrm{i}} \\ 0 & 0 & 0 & 1\end{array}\right], \quad \boldsymbol{M}_{\text {lens }}=\left[\begin{array}{cccc}1 & 0 & 0 & 0 \\ -\frac{1}{f} & 1 & 0 & 0 \\ 0 & 0 & 1 & 0 \\ 0 & 0 & -\frac{1}{f} & 1\end{array}\right]$,

$\boldsymbol{M}_{\mathrm{a}}=\left[\begin{array}{cccc}1 & d_{\mathrm{a}} & 0 & 0 \\ 0 & 1 & 0 & 0 \\ 0 & 0 & 1 & d_{\mathrm{a}} \\ 0 & 0 & 0 & 1\end{array}\right], \quad \boldsymbol{M}_{\mathrm{r}}=\left[\begin{array}{cccc}1 & 0 & 0 & 0 \\ \frac{n_{1}-n_{2}}{n_{2} R} & \frac{n_{1}}{n_{2}} & 0 & 0 \\ 0 & 0 & 1 & 0 \\ 0 & 0 & 0 & \frac{n_{1}}{n_{2}}\end{array}\right]$,

$\boldsymbol{M}_{\mathrm{o}}=\left[\begin{array}{llll}1 & d_{\mathrm{o}} & 0 & 0 \\ 0 & 1 & 0 & 0 \\ 0 & 0 & 1 & d_{\mathrm{o}} \\ 0 & 0 & 0 & 1\end{array}\right]$.

In order to hold the object-image relationship, the entries $S_{12}$ and $S_{34}$ of the transfer matrix $S$ must be equal to 0 , that is

$$
\left\{\begin{array}{l}
s_{12}=\frac{n_{1}\left(d_{\mathrm{i}}-d_{\mathrm{a}}\left(\frac{d_{\mathrm{i}}}{f}-1\right)\right)}{n_{2}}+ \\
d_{\mathrm{o}}\left(\frac{\left(n_{1}-n_{2}\right)\left(d_{\mathrm{i}}-d_{\mathrm{a}}\left(\frac{d_{\mathrm{i}}}{f}-1\right)\right)}{R n_{2}}-\frac{d_{\mathrm{i}}}{f}+1\right)=0 \\
s_{34}=\frac{n_{1}\left(d_{\mathrm{i}}-d_{\mathrm{a}}\left(\frac{d_{\mathrm{i}}}{f}-1\right)\right)}{n_{2}}-d_{\mathrm{o}}\left(\frac{d_{\mathrm{i}}}{f}-1\right)=0
\end{array}\right.
$$


$S_{12}$ denotes the object-image relationship in the $x z$ plane. $S_{34}$ denotes the object-image relationship in the $y z$ plane. It can be found from Eq. (8) that $S_{12}$ is related to the radius $R$ of the outer surface and the thickness $d_{\mathrm{o}}$ of a glass ring, while $S_{34}$ is only related to the glass ring thickness $d_{\mathrm{o}}$, other than the position of microscope and CCD. If it is assumed that the air distance $d_{\mathrm{a}}$ (namely the position of microscope) is a variable, and the other parameters are fixed, the set of Eq. (8) has no solution. This means that the object-image relationship cannot hold in the $x z$ and the $y z$ planes simultaneously. Assuming $d_{\mathrm{ax}}$ and $d_{\mathrm{ay}}$ are the required air distances that satisfy the object-image relationship in the $x z$ and the $y z$ planes, respectively, $d_{\mathrm{ax}}$ and $d_{\mathrm{ay}}$ can be obtained from Eq. (8) as follows:

$$
\begin{aligned}
& \left\{\begin{array}{l}
d_{\mathrm{a} x}=-\frac{f d_{\mathrm{i}}}{f-d_{\mathrm{i}}}-\frac{R n_{2} d_{\mathrm{o}}}{R n_{1}+d_{\mathrm{o}}\left(n_{1}-n_{2}\right)} \\
d_{\mathrm{a} y}=-\frac{f d_{\mathrm{i}}}{f-d_{\mathrm{i}}}-\frac{n_{2} d_{\mathrm{o}}}{n_{1}}
\end{array}\right. \\
& d_{\mathrm{ax}}-d_{\mathrm{a} y}=\frac{n_{2} d_{\mathrm{o}}^{2}\left(n_{1}-n_{2}\right)}{n_{1}\left(R n_{1}+d_{\mathrm{o}}\left(n_{1}-n_{2}\right)\right)}
\end{aligned}
$$

If $\left|d_{\mathrm{ax}}-d_{\mathrm{ay}}\right|$ is less than the depth of the field, the camera can still obtain clear images. However, a microscope has to be used in the test rig to magnify the interference image in the contact region in order to analyze the film thickness. The depth of the field of the microscope is usually very shallow, particularly with higher magnification. Table 1 shows the parameters used in the test rig in Fig. 1, and $\left|d_{a x}-d_{a y}\right|$ is obtained using Eq. (10), where the depth of field is taken using Zoom 6000 performance specifications of NAVITAR. It can be found that the depth of field of the image capturing system is much less than $\left|d_{\mathrm{ax}}-d_{\mathrm{ay}}\right|$, which means that the microscope cannot be in-focus in the $x z$ and the $y z$ planes simultaneously, resulting in

Table 1 Actual parameters used in the test rig and $\left|d_{\mathrm{ax}}-d_{\mathrm{ay}}\right|$.

\begin{tabular}{cccccc}
\hline $\begin{array}{c}d_{\mathrm{o}} \\
(\mathrm{mm})\end{array}$ & $n_{1}$ & $n_{2}$ & $\begin{array}{c}R \\
(\mathrm{~mm})\end{array}$ & $\begin{array}{c}\left|d_{\mathrm{ax}}-d_{\mathrm{ay}}\right| \\
(\mathrm{mm})\end{array}$ & $\begin{array}{c}\text { Depth of field } \\
(\mathrm{mm})\end{array}$ \\
\hline 13.58 & 1.5 & 1 & -65 & 0.678 & Less than 0.02 \\
\hline
\end{tabular}

interference images blurring and ghosting as shown in Fig. 2.

The above analysis of the blurring and ghosting of the interference image is schematically shown in Fig. 5. To easily compare the imaging of the system in the $x z$ plane and the $y z$ plane, the optical paths in both $x z$ and $y z$ planes are drawn in the same diagram. Based on Snell's law, the optical path in the $y z$ plane refracts more outwards than that in the $x z$ plane at the outer surface of the ring. Thus, as shown in Fig. 5(a), rays from object point focus on the CCD in the $x$ direction, while behind the CCD in the $y$ direction when $d_{\mathrm{a}}$ is equal to $d_{\mathrm{ax}}$. Therefore, the interference fringe is clear in the $x$ direction, and blurry in the $y$ direction. If $d_{\mathrm{a}}$ is equal to $d_{\mathrm{ay}}$, rays from the object point focus on the CCD in the $y$-direction, while in front of the CCD in $x$ direction. Therefore the interference fringe is clear in the $y$ direction and blurry in the $x$ direction, as shown in Fig. 5(b). When $d_{\mathrm{a}}$ is in between $d_{\mathrm{a} y}$ and $d_{\mathrm{a} x}$, the interference fringe will be blurry in both directions as the rays will not focus on the CCD in both directions, as shown in Fig. 5(c). In Fig. 2, the images from left to right were captured by the test rig while increasing the observation distance, which corresponds to the increase of $d_{\mathrm{a}}$ from $d_{\mathrm{ax}}$ to $d_{\mathrm{a} y}$. Therefore, the blurring and ghosting of the interference image are consistent with the results of the matrix theory. Such interference images cannot be used to determine the oil film thickness because the interference fringes are not adequately sharp and clear. The measurement must be taken after improving the test rig to obtain high-quality interference images.

Based on the above optical analysis, in order to obtain clear interference images, the optical path must be corrected according to the formulation of the optical system. Thus, a cylindrical lens is introduced to adjust the different refractions in the $x$ and $y$ directions at the outer cylindrical surface of ring, as shown in Fig. 6 . The axis of the cylindrical lens is parallel to the axis of the glass ring, and intersects perpendicularly with the optical axis of the system. When a light arrives at the cylindrical lens, it first refracts on the cylindrical surface, then travels in the cylindrical lens, and finally refracts at the flat surface leaving the cylindrical lens. Thus, the optical transfer matrix of this cylindrical 
(a)

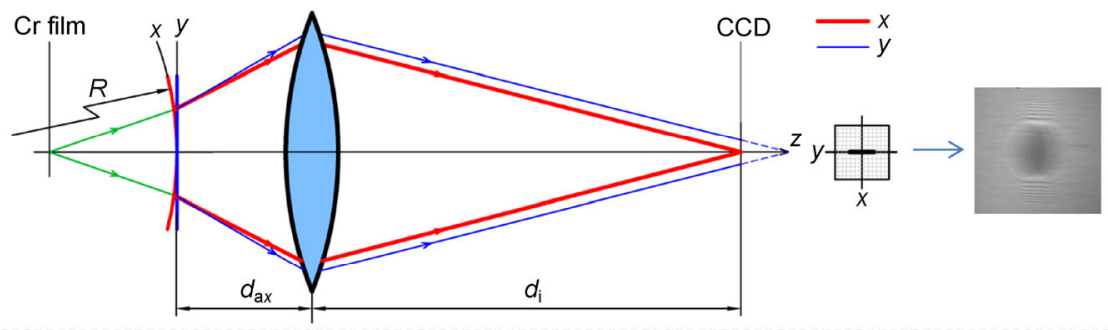

(b)

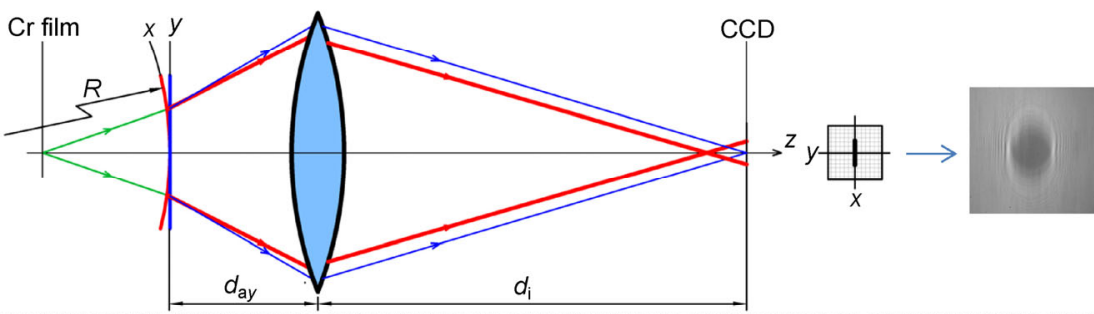

(c)

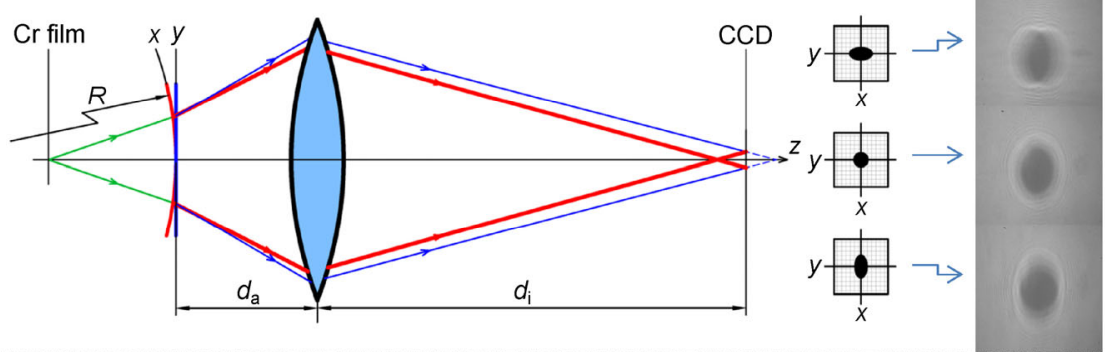

Fig. 5 Optical path diagram of an optical system showing different images of a point against $d_{\mathrm{a} \text {. }}$

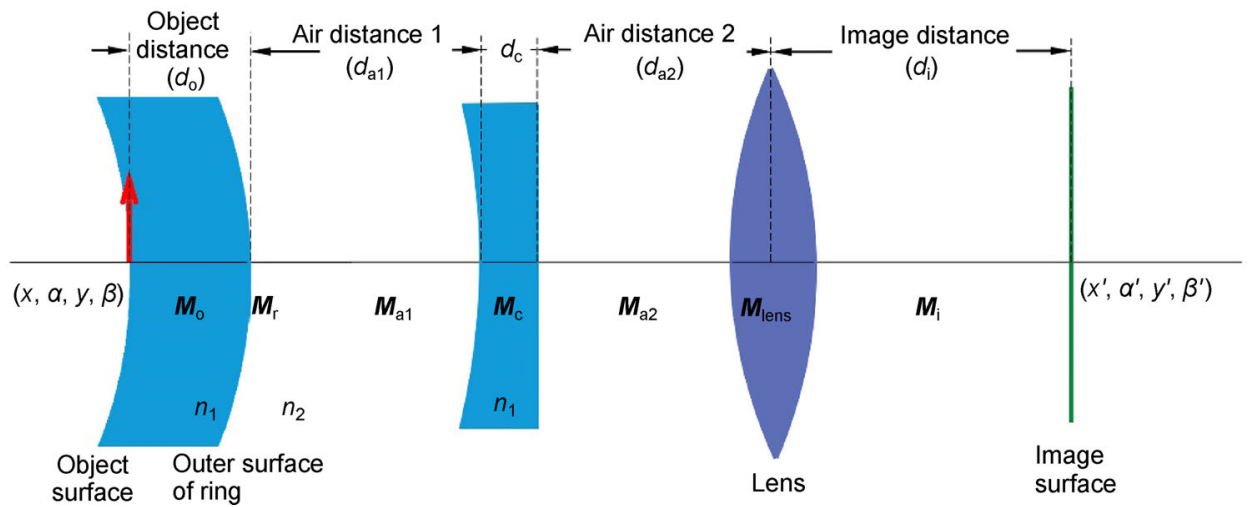

Fig. 6 Corrected optical system.

lens can be written as follows:

$$
\boldsymbol{M}_{\mathrm{c}}=\left[\begin{array}{cccc}
1 & 0 & 0 & 0 \\
0 & \frac{n_{1}}{n_{2}} & 0 & 0 \\
0 & 0 & 1 & 0 \\
0 & 0 & 0 & \frac{n_{1}}{n_{2}}
\end{array}\right]\left[\begin{array}{cccc}
1 & d_{\mathrm{c}} & 0 & 0 \\
0 & 1 & 0 & 0 \\
0 & 0 & 1 & d_{\mathrm{c}} \\
0 & 0 & 0 & 1
\end{array}\right]\left[\begin{array}{cccc}
1 & 0 & 0 & 0 \\
\frac{n_{2}-n_{1}}{n_{1} R_{\mathrm{c}}} & \frac{n_{2}}{n_{1}} & 0 & 0 \\
0 & 0 & 1 & 0 \\
0 & 0 & 0 & \frac{n_{2}}{n_{1}}
\end{array}\right]
$$

Then, the optical transfer matrix of the corrected optical system is as follows:

$$
\boldsymbol{C}=\boldsymbol{M}_{\mathrm{i}} \boldsymbol{M}_{\text {lens }} \boldsymbol{M}_{\mathrm{a} 2} \boldsymbol{M}_{\mathrm{c}} \boldsymbol{M}_{\mathrm{a} 1} \boldsymbol{M}_{\mathrm{r}} \boldsymbol{M}_{\mathrm{o}}
$$

where $\boldsymbol{M}_{\mathrm{a} 1}$ is the transfer matrix when light travels in the air between the glass ring and the cylindrical lens, while $M_{\mathrm{a} 2}$ is the transfer matrix when light travels in the air between the cylindrical lens and the lens. They are depicted as follows: 


$$
\boldsymbol{M}_{\mathrm{a} 1}=\left[\begin{array}{cccc}
1 & d_{\mathrm{a} 1} & 0 & 0 \\
0 & 1 & 0 & 0 \\
0 & 0 & 1 & d_{\mathrm{a} 1} \\
0 & 0 & 0 & 1
\end{array}\right], \quad \boldsymbol{M}_{\mathrm{a} 2}=\left[\begin{array}{cccc}
1 & d_{\mathrm{a} 2} & 0 & 0 \\
0 & 1 & 0 & 0 \\
0 & 0 & 1 & d_{\mathrm{a} 2} \\
0 & 0 & 0 & 1
\end{array}\right]
$$

where $d_{\mathrm{a} 1}$ is the distance between the glass ring and the cylindrical lens, and $d_{\mathrm{a} 2}$ is the distance between the cylindrical lens and the lens.

Based on the object-image relationship, $d_{\mathrm{a} 1}$ and $d_{\mathrm{a} 2}$ can be obtained through Eq. (12) in the $x$ direction and the $y$ direction as follows:

$$
\left\{\begin{array}{l}
d_{\mathrm{a} 1}=-\frac{n_{2} d_{\mathrm{o}}\left(2 R n_{1}+n_{1} d_{\mathrm{o}}-n_{2} d_{\mathrm{o}}+A\right)}{2 n_{1}\left(R n_{1}+n_{1} d_{\mathrm{o}}-n_{2} d_{\mathrm{o}}\right)} \\
d_{\mathrm{a} 2}=-\frac{f d_{\mathrm{i}}}{f-d_{\mathrm{i}}}-\frac{n_{2}}{n_{1}}\left(d_{\mathrm{c}}+d_{\mathrm{o}}\right)-d_{\mathrm{a} 1}
\end{array}\right.
$$

where

$A=\sqrt{n_{1}^{2} d_{\mathrm{o}}^{2}+4 R_{\mathrm{c}} n_{1}^{2} d_{\mathrm{o}}+4 R R_{\mathrm{c}} n_{1}^{2}-2 n_{1} n_{2} d_{\mathrm{o}}^{2}-4 R_{\mathrm{c}} n_{1} n_{2} d_{\mathrm{o}}+n_{2}^{2} d_{\mathrm{o}}^{2}}$.

According to Eq. (13), $d_{\mathrm{a} 1}$ depends only on the glass ring and the cylindrical lens, while $d_{\mathrm{a} 2}$ depends on the glass ring, cylindrical lens, and microscope. As Fig. 7 shows, using the parameters in Table $2, d_{\mathrm{a} 1}$ increases with increasing radius of the cylindrical lens used, while $d_{\mathrm{a} 2}$ decreases. To correct the light deviation at the outer surface of the ring, the radius of the cylindrical lens $R_{\mathrm{c}}$ must be larger than or equal to the outer radius

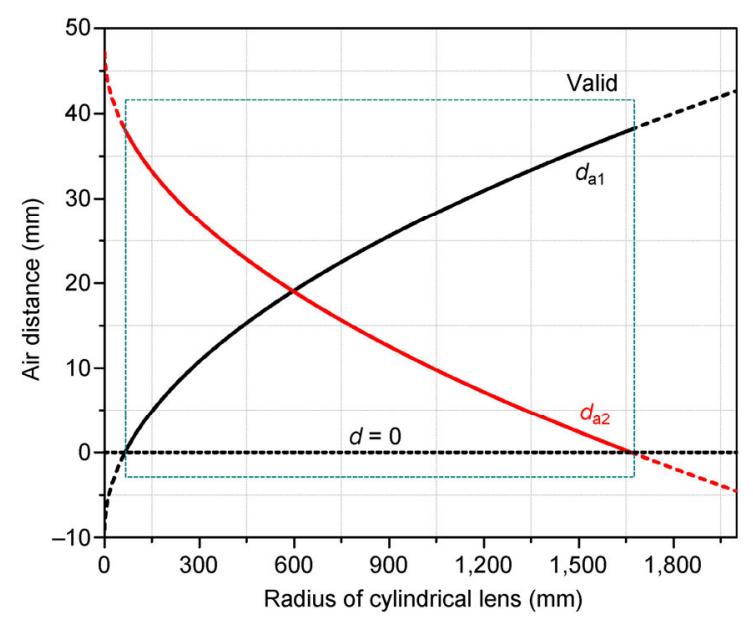

Fig. $7 d_{\mathrm{a} 1}$ and $d_{\mathrm{a} 2}$ vary with the radius of cylindrical lens.

Table 2 Structure parameters used in Fig. 7.

\begin{tabular}{cccccccc}
\hline $\begin{array}{c}d_{\mathrm{o}} \\
(\mathrm{mm})\end{array}$ & $n_{1}$ & $n_{2}$ & $\begin{array}{c}R \\
(\mathrm{~mm})\end{array}$ & $\begin{array}{c}d_{\mathrm{i}} \\
(\mathrm{mm})\end{array}$ & $\begin{array}{c}d_{\mathrm{c}} \\
(\mathrm{mm})\end{array}$ & $\begin{array}{c}f \\
(\mathrm{~mm})\end{array}$ & $\begin{array}{c}-R_{\mathrm{c}} \\
(\mathrm{mm})\end{array}$ \\
\hline 13.58 & 1.5 & 1 & -65 & 22 & 0 & 15 & $0 \sim 1,500$ \\
\hline
\end{tabular}

of ring $R$, and smaller than the value limited by $d_{\mathrm{a} 2}$ in order to avoid interfering with the microscope objective lens. Thus, by mounting a cylindrical lens with the proper distance described above, the optical system can focus the interference images on the CCD simultaneously in the $x$ direction and $y$ direction. Thereupon, analyzable interference images can be obtained by this corrected optical system.

\section{Discussion}

For an optical system with a cylindrical interface, where unexpected optical deviation occurs, a coupled cylindrical lens with the same diameter can avoid its position adjustment by closely fitting with the interface [13]. However, in the current ball-on-ring test rig, the glass ring rotates at a very high speed, and thus, the coupled cylindrical lens cannot be fitted closely on the ring due to friction, damage of the optical surface, and interfering with the fixture. According to the result for $d_{\mathrm{a} 1}$ given in Fig. 7, a concave cylindrical lens with a diameter of approximately between $200 \mathrm{~mm}$ $1,600 \mathrm{~mm}$ could be used to overcome the problem of fuzzy images and avoid interfering with other elements in the original ball-on-ring test rig. So instead, a concave cylindrical lens of $1,000 \mathrm{~mm}$ in diameter is mounted above the glass ring by a distance of about $30 \mathrm{~mm}$.

The corrected ball-on-ring measurement system was able to precisely capture the optical interference images at the ball-on-ring contact region. In order to check the validation of the proposed approach, the test rig was improved with the updated optical imaging system, and preliminary experiments were conducted under a tractive rolling condition, where the steel ball was driven by the glass ring. Figure 8 shows the images captured by a monochrome CCD with a coherent light source in the same incidence condition as Fig. 2. Figures 8(a) and 8(d) show the interference images captured by the uncorrected image system. Figures $8(\mathrm{~b})$ and $8(\mathrm{e})$ show the interference images captured by the uncorrected imaging system, but with small aperture diaphragm. Figures 8(c) and $8(\mathrm{f})$ present the interference images captured by the corrected imaging system. Figure 9 shows the optical intensities of the images in Fig. 8 at the centerline along the motion direction and the film profiles 


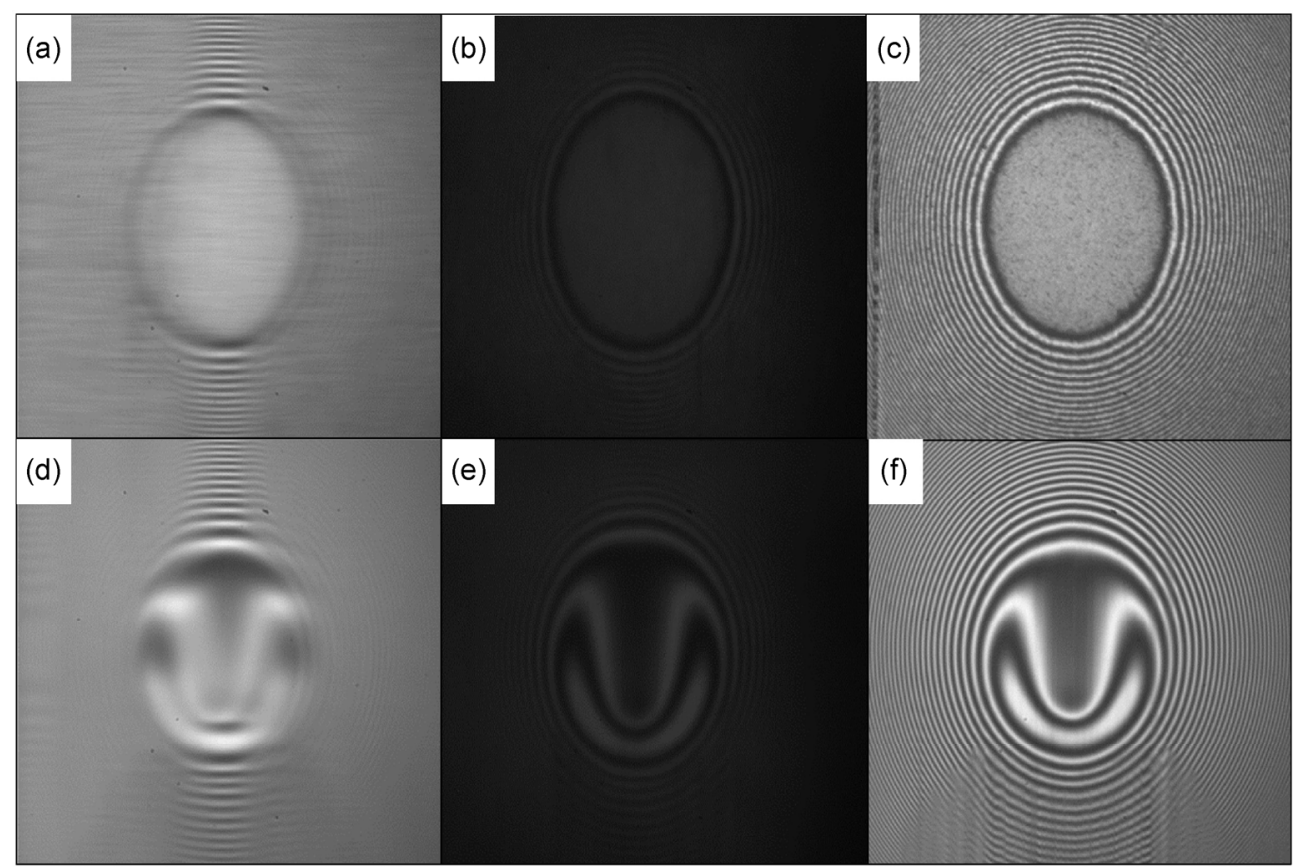

Fig. 8 Interference images captured by: $(a, d)$ uncorrected system; (b, e) uncorrected system with small aperture diaphragm; (c, f) corrected system, at rest $(\mathrm{a}, \mathrm{b}, \mathrm{c})$ and at the speed $5 \mathrm{~m} / \mathrm{s}(\mathrm{d}, \mathrm{e}, \mathrm{f})$.

corresponding to these images. Figures 8(c) and 8(f), captured by the corrected system clearly show the change in the interference fringes over the entire contact region, as they have high saturation and definition. This validates the effectiveness of the proposed ballon-ring test rig. Figures 8(a) and 8(d), captured by the uncorrected test rig, are only in-focus in a very narrow field. Although they also have good saturation, most regions of the images have gray level deviations compared to Figs. 8(c) and 8(f), resulting in the different film thicknesses shown in Fig. 9. Using a smaller aperture helped to reduce the cone angle of the rays, resulting in a clear image in the center region, as shown in Figs. 8(b) and 8(e). However, the narrower the aperture, the darker the image becomes when the other factors are kept constant. Meanwhile, the length of the contact radius in the $x$ direction is magnified because the cylindrical surface refracts the rays in the $y z$ plane through larger angles (see Eq. (6) and Fig. 5), while aperture limits the light admission. The aberration of the contact radius can also be observed in Fig. 9.

The images captured by the corrected system have an extra magnification in the $x$ direction due to the cylindrical shape of the glass ring and the cylindrical lens, other than the magnification of the microscope.
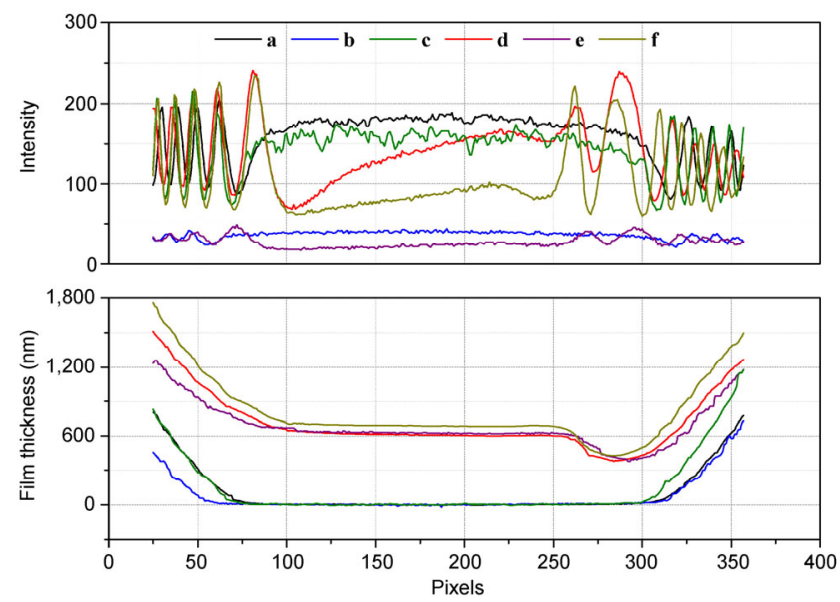

Fig. 9 Optical intensities of the images in Fig. 8 at the centerline along the motion direction and film profiles corresponding to each image.

In an optical system of centered lenses, the lateral magnifications are $m_{11}$ and $m_{33}$ in Eq. (1). In this paper, as shown in Fig. 10, the corrected system has different lateral magnifications in the $x$ and $y$ directions because of the radii of the ring and the cylindrical lens used.

It should be noted that, the blurring phenomenon in the interferogram can be eliminated by the usage of the concave cylindrical lens, and the quality of the interference image can be further improved by 


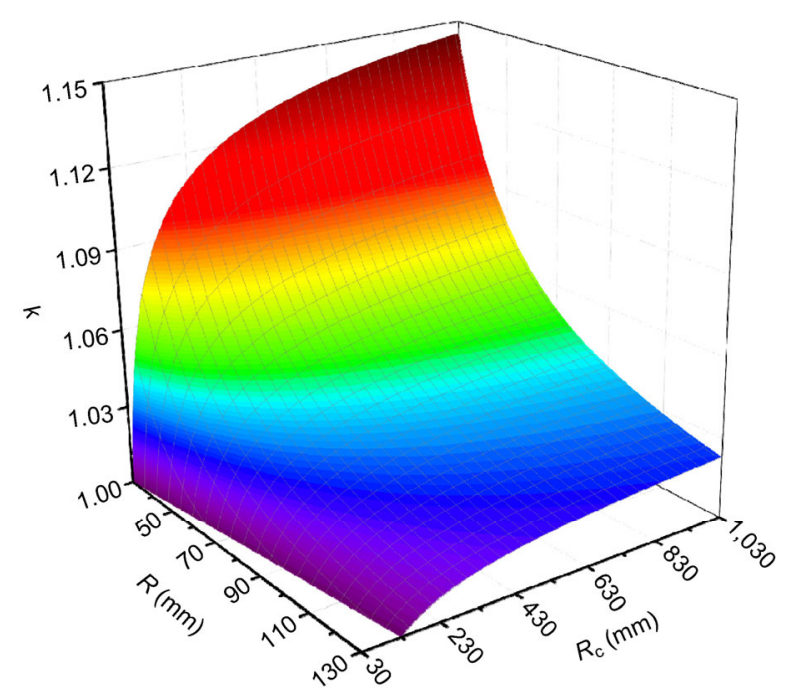

Fig. 10 Ratio between lateral magnification in the $x$ and $y$ directions in the corrected system $\left(n_{1}=1.5, n_{2}=1.0, d_{0}=13.58\right)$.

choosing appropriate parameters for the Cr-coated film thickness, coating material and so on, which can be referred in Ref. [14].

\section{Conclusion}

The development of modern equipment continuously increases the demand of the rolling bearing, for which the lubrication plays a very important role, particularly at high-speed/heavy-load conditions. In order to explore the lubrication behavior of highspeed ball bearing, this paper presented a new test rig for the optical measurement of oil film thickness based on a ball-on-ring mode. This closely imitated the contact between the ball and the outer ring in a ball bearing. The following conclusions can be drawn from the results of this study.

Owing to the unexpected optical refraction at the outer cylindrical surface of the ring, the interference images were blurry and exhibited ghosting, which is not suitable for determining oil film thickness.

An optical model was developed to analyze the measurement system of the ball-on-ring mode. Based on the optical analysis, the reason for the haziness of the interference image was explored. An improved measurement was proposed to obtain high-quality interference images to successfully investigate the lubrication behaviors of high-speed rolling bearings.

\section{Acknowledgement}

The work was supported by the National Natural Science Foundation of China (Nos. 51675046 and 51275045) and the National Key Basic Research (973) Program of China (No. 2011CB706602).

Open Access: The articles published in this journal are distributed under the terms of the Creative Commons Attribution 4.0 International License (http:// creativecommons.org/licenses/by/4.0/), which permits unrestricted use, distribution, and reproduction in any medium, provided you give appropriate credit to the original author(s) and the source, provide a link to the Creative Commons license, and indicate if changes were made.

\section{References}

[1] Gohar R, Cameron A. Optical measurement of oil film thickness under elastohydrodynamic lubrication. Nature 200: 458-459 (1963)

[2] Gohar R, Cameron A. The mapping of elastohydrodynamic contacts. Tribol Trans 10: 215-225 (1967)

[3] Johnston G J, Wayte R, Spikes H A. The measurement and study of very thin lubricant films in concentrated contacts. Tribol Trans 34: 187-194 (1991)

[4] Cann P M, Spikes H A, Hutchinson J. The development of a spacer layer imaging method (slim) for mapping elastohydrodynamic contacts. Tribol Trans 39: 915-921 (1996)

[5] Spikes H A, Cann P M. The development and application of the spacer layer imaging method for measuring lubricant film thickness. Proc IMechE, Part J: J Eng Tribol 215: 261-277 (2001)

[6] Hartl M, Křupka I, Liška M. Elastohydrodynamic film thickness mapping by computer differential colorimetry. Tribol Trans 42: 361-368 (1999)

[7] Hartl M, Krupka I, Poliscuk R, Liska M, Molimard J, Querry M, Vergne P. Thin film colorimetric interferometry. Tribol Trans 44: 270-276 (2001)

[8] Luo J B, Wen S Z, Huang P. Thin film lubrication. Part I. Study on the transition between EHL and thin film lubrication using a relative optical interference intensity technique. Wear 194: 107-115 (1996)

[9] Guo F, Wong P L. A multi-beam intensity-based approach for lubricant film measurements in non-conformal contacts. Proc IMechE, Part J: J Eng Tribol 216: 281-291 (2002) 
[10] Hili J, Olver A V, Edwards S, Jacobs L. Experimental investigation of elastohydrodynamic (EHD) film thickness behavior at high speeds. Tribol Trans 53: 658-666 (2010)

[11] Liang H, Guo D, Luo J B. Experimental investigation of lubrication film starvation of polyalphaolefin oil at high speeds. Tribol Lett 56: 491-500 (2014)

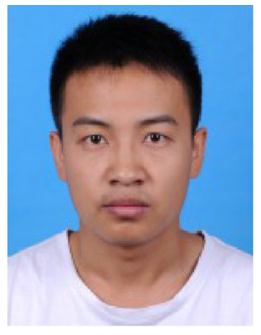

Yaoguang ZHANG. He received his bachelor degree in Automation School in 2014 from Beijing University of Posts and Telecommunications, Beijing, China. Now he is a Ph.D. student in School of Mechanical

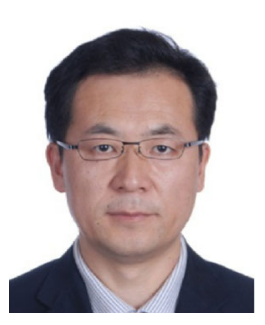

Wenzhong WANG. He received his Ph.D. degree in mechanical engineering from Tsinghua University, China, in 2003, and then he worked in State Key Laboratory of Tribology as a postdoc research fellow. He joined School of Mechanical Engineering

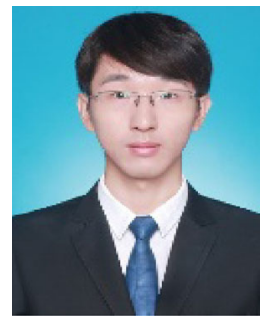

Shengguang ZHANG. He received his bachelor degree in School of Mechanical Engineering in 2011 from Beijing Institute of Technology, Beijing, China. Now he is a Ph.D. student at the same university. His research focuses on the modeling

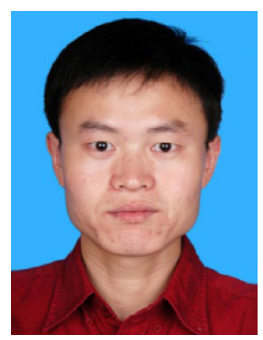

Ziqang ZHAO. He received his Ph.D. degree in mechanical engineering from Beijing Institute of Technology, China, in 2009. He joined School of Mechanical Engineering at Beijing Institute of Technology from 2009.
[12] Gerrard A, Burch J M. Introduction to Matrix Methods in Optics. London: John Wiley \& Sons, 1975.

[13] Kirk M T. Hydrodynamic lubrication of 'perspex'. Nature 194: 965-966 (1962)

[14] Fu Z, Guo F, Wong P L. Theoretical study on the interferometry of thin EHL film measurement. Tribol Lett 31: 57-65 (2008)

Engineering in Beijing Institute of Technology, Beijing, China. His current research interests include elastohydrodynamic lubrication film thickness measurement and lubricant performance considering limiting shear stress at very high speed.

at Beijing Institute of Technology from 2006. His current position is a professor and director of Department of Machine Design. His research areas cover modeling of lubricated contact problem, contact model for inhomogeneous and function gradient materials, dynamics of rolling bearing, and thermal analysis.

analysis of lubricated contact problem considering the effect of material inhomogeneity. His research interests also include the dynamic modeling analysis of rolling bearings and the measurement of lubrication film thickness by optical interferometry method.

His current position is a lecturer. His research areas cover dynamic theory and experiment of gear transmission system and rolling bearing, numerical simulation of lubrication, thermal analysis, and mechanical design and so on. 